\title{
Estudos de custo sobre terapia dialítica no mundo: uma re- visão sistemática e uma abordagem histórica
}

\section{HUevista}

Cost studies on dialysis therapy in the world: a systematic review and a

historical approach

Alyne Schreider Manoel Francisco de Souza Neto 2 Juliana Paula Pereira ${ }^{1}$ Natália Maria da Silva Fernandes ${ }^{1,3}$

${ }^{1}$ Pós-graduação em Saúde, Universidade Federal de Juiz de Fora, Brasil.

${ }^{2}$ Graduação em Estatística, Universidade Federal de Juiz de Fora, Brasil.

${ }^{3}$ Departamento de Clínica Médica, Faculdade de Medicina, Universidade Federal de Juiz de Fora, Brasil.

\section{Natália Fernandes}

Rua Jamil Altaf, 132, Vale do Ipê, Juiz de Fora, Minas Gerais

CEP: $36085-380$

७ nataliafernandes02@gmail.com

\section{RESUMO}

Introdução: A doença renal afeta 850 milhões de pessoas no mundo e é uma das principais contribuintes para a carga global de doenças não transmissíveis (DNT). Projeta-se que até 2040 seja a quinta causa de anos de vida perdidos. Objetivo: Avaliar onde (geograficamente) e como estão sendo feitos os estudos de custo em terapia dialítica no mundo. Material e métodos: Foi realizada nos dias 26/06/2017, 10/07/2017 e 30/09/2018 uma busca com os unitermos "cost and cost analysis", "health expenditures" and "peritoneal dialysis", os mesmos unitermos e "renal dialysis" nas bases Pubmed, Scielo e EmBase e foram encontrados 1796 artigos, destes foram selecionados 124. Acrescentou-se análise no Google Acadêmico. Ao final, analisou-se 125 artigos. Os critérios de inclusão dos artigos foram terem como escopo do estudo o custo, utilizando metodologias diversas como custo, custo total, custo efetividade, custo utilidade, custo benefício, custo minimização, gasto, impacto econômico. A perspectiva poderia ser do pagador e do prestador de serviço. Resultados: Observou-se um maior número de publicações a partir do ano 2000. Com relação ao desenho dos estudos, a maioria apresentou metodologia de difícil reprodutibilidade, utilizando conceitos variados e métodos diversos. Há uma grande variabilidade do custo entre os países e até mesmo entre os centros de diálise. Em linhas gerais, em países de alta renda, a DPAC (diálise peritoneal ambulatorial contínua) e a DPA (diálise peritoneal automatizada) apresentam menor custo quando comparadas à HD (hemodiálise). No Brasil a maioria dos estudos mostra HD apresentando menor custo que DPAC e DPA. Os desenhos são diversos e mostram, em geral, a perspectiva do pagador. Conclusão: Estudos de custos são recentes na literatura, incluem métodos diversos e a descrição dos resultados não é padronizada. Esperamos progredir para uma discussão da saúde baseada em valor.

Palavras-chaves: Custos e Análise de Custo; Gastos em Saúde; Diálise Peritoneal; Diálise Renal.

\section{ABSTRACT}

Introduction: Kidney disease affects 850 million people worldwide and is a major contributor to the global burden of noncommunicable diseases (NCDs). By 2040 it is projected to be the fifth cause of lost years of life. Objective: The aim of this review is to evaluate where (geographically) and how cost studies on dialysis therapy are being done in the world. Material and methods: A search was carried out on 06/26/2017, 07/10/2017 and 09/30/2018 with the keywords "cost and cost analysis", "health expenditures" and "peritoneal dialysis". The same keywords and "renal dialysis" in Pubmed, Scielo and EmBase databases and 1796 articles were found, of which 124 were selected. We added analysis in academic google. At the end we analyzed 125 articles. The inclusion criteria of the articles were the scope of the study cost, using various methodologies such as cost, total cost, cost effectiveness, cost utility, cost benefit, cost minimization, expense, economic impact. The perspective could be from the payer and the service provider. Results: We observed a greater number of publications from the year 2000. Regarding the design of the studies, most presented a difficult to reproduce methodology with different concepts and different method. There were great variability across countries and even between dialysis centers. Overall, in high-income countries, continuous ambulatory peritoneal dialysis (CAPD) and automated peritoneal dialysis (APD) are less expensive than HD (hemodialysis). In Brazil most studies show HD presenting lower cost than CAPD and APD. Conclusion: Cost studies are recent in the literature, include several methods and the description of the results is not standardized. The designs are diverse and generally show the perspective of the payer. We hope to progress to a value-based health discussion.

Key-words: Costs and Cost Analyses; Health Expenditures; Peritoneal Dialysis; Renal Dialysis. 


\section{INTRODUÇÃO}

A Sociedade Internacional de Nefrologia, em recente publicação, enfatiza que a doença renal afeta 850 milhões de pessoas no mundo e é uma das principais contribuintes para a carga global de doenças não transmissíveis (DNT) como causa e consequência de outras principais DNT e a principal causa de gastos catastróficos em saúde. Se não for abordada, projeta-se que até 2040 seja a quinta causa mais comum de anos de vida perdidos. ${ }^{1}$

A princípio, a saúde deveria ser um bem acima da preocupação econômica. Idealmente, a saúde não tem preço. Não podendo viver essa utopia, a realidade da limitação dos recursos nos obriga a associar à área da saúde estudos e análises típicas da área econômica, em particular na gestão de recursos escassos e tomada de decisões.

No Brasil, uma análise demográfica criteriosa vai destacar mudanças importantes do perfil da sociedade com transformações significativas na pirâmide populacional nas últimas décadas. ${ }^{2} \mathrm{O}$ envelhecimento da população traz, na sua esteira, impactos nos gastos com a saúde, não só pela incorporação de novas tecnologias, como também pela necessidade de tratamentos de doenças crônicas, típicas de idades mais avançadas. No mundo, a mortalidade infantil diminuiu, a expectativa de vida aumentou, a cobertura vacinal foi ampliada e algumas doenças erradicadas. ${ }^{3}$ Tudo isso traz consequências de ordem econômica.

As mudanças tecnológicas em saúde ocorrem nos medicamentos, nos equipamentos, nas cirurgias e procedimentos, na estrutura organizacional das instituições de saúde, na informação e educação e também nos programas governamentais de assistência à população. Decisões administrativas e políticas na gestão dos recursos da saúde esbarram nas dificuldades impostas pela limitação desses recursos e a crescente demanda do sistema de saúde público.

A alocação eficiente de recursos torna-se uma questão de grande importância, não permitindo decisões baseadas em intuição ou critérios meramente políticos. Torna-se necessário assegurar a sobrevivência de programas essenciais, reexame de benefícios e a implantação de programas que se mostrarem críticos às novas condições da população e ameaças à saúde. Em um cenário mundial em nefrologia, projeta-se que em 2030 teremos 5,4 milhões de pacientes em terapia dialítica e 14 milhões com necessidade dialítica, porém sem acesso às terapias disponíveis. ${ }^{3}$ Para que consigamos melhorar esta previsão, devemos ter estudos de custo adequados a cada região e que possam ser replicados.

O objetivo desta revisão é avaliar onde (geograficamente) e como estão sendo feitos os estudos de custo em terapia dialítica no mundo.

\section{MATERIAL E MÉTODOS}

Foi realizada, no dia 26 de junho de 2017, uma busca com os unitermos "cost and cost analysis" and "peritoneal dialysis" nas bases Pubmed, Scielo e EmBase e foram encontrados 339 artigos, destes, foram selecionados 52 que apresentavam resumos na língua inglesa, portuguesa, espanhola, francesa ou italiana. No dia 10 de julho de 2017, foi feito uma busca nas mesmas bases utilizando os unitermos "health expenditures" e "peritoneal dialysis", foram encontrados sete artigos e foram selecionados três que apresentavam resumo em inglês ou português, dos sete dois estavam duplicados e dois foram selecionados. No dia 30 de setembro de 2018, foi realizada uma busca com os unitermos "cost and cost analysis" and "renal dialysis"; "health expediture" and "renal dialysis"; foi encontrado um total de 1450 artigos, foram selecionados 112 artigos e destes, 18 já estavam incluídos na pesquisa anterior, ao rever os resumos observamos que 24 não preenchiam os critérios de inclusão, restando 70 artigos. Ao final acrescentamos às palavras chave citadas o unitermo "Brazil" e fizemos nova busca nas bases citadas. Acrescentamos ainda análise na base Google Acadêmico e encontramos mais um artigo não citado anteriormente com os unitermos citados. Ao final analisamos 125 artigos. A pesquisa foi realizada por dois pesquisadores independentes.

Os critérios de inclusão dos artigos foram terem como escopo do estudo custo, utilizando metodologias diversas como custo direto, custo indireto, custo total, custo efetividade, custo utilidade, custo benefício, custo minimização, gasto, impacto econômico. A perspectiva poderia ser do pagador e do prestador de serviço. O desenho do estudo poderia ser transversal, de coorte ou simulação, portanto o horizonte temporal poderia variar. A população avaliada foi de pacientes com doença renal crônica (DRC). Todas as modalidades de diálise peritoneal (DP) foram incluídas: diálise peritoneal ambulatorial contínua (DPAC), diálise peritoneal automatizada (DPA) e diálise peritoneal assistida. Foram incluídas as modalidades de hemodiálise (HD) padrão, hemodiálise de alta dose, hemodiálise noturna além de hemodiafiltração, realizadas de forma hospitalar, domiciliar ou em centros satélites. Estudos comparativos que incluíram DRC pré dialítica e transplante renal, desde que no contexto das terapias dialíticas, também foram incluídos. Os critérios de não inclusão foram não ter custo em nenhuma das formas citadas como escopo do estudo, estudos com insuficiência renal aguda ou não apresentar resumo nas línguas inglesa, portuguesa, espanhola, francesa ou italiana.

A seguir descreveremos os principais achados dos estudos analisados da forma mais cronológica e histórica possível. 


\section{RESULTADOS E DISCUSSÃO}

Observamos, na figura 1, um maior número de publicações a partir do ano 2000 . Com relação ao desenho dos estudos, a maioria apresentou metodologia de difícil reprodutibilidade, utilizando conceitos diversos para definição de custos e diversas formas de descrição dos resultados (figura 2 ).

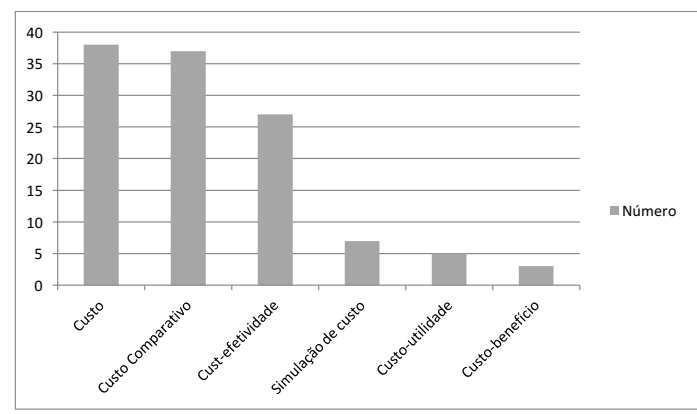

Figura 1: Número de artigos publicados por década.

Na figura 3, observamos que a maior parte dos estudos foi realizada em países de alta e média renda, resultado semelhante ao observado no Atlas Global de Saúde Renal publicado pela Sociedade Internacional de Nefrologia em 2019. ${ }^{3} \mathrm{Na}$ América do Sul, encontramos estudos apenas no Brasil e no Chile.

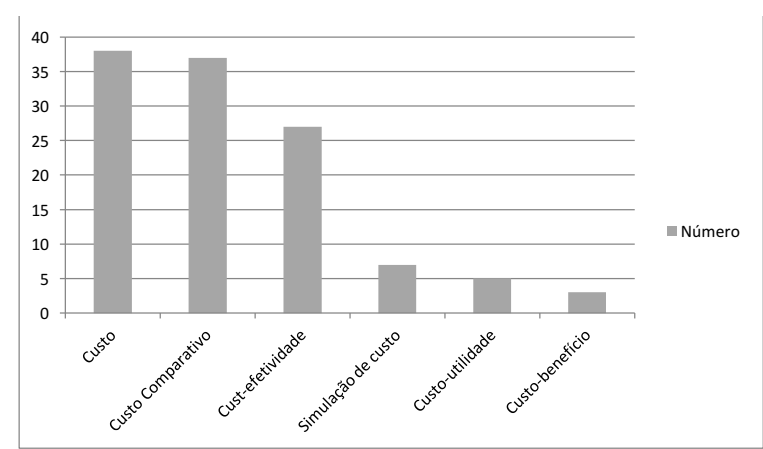

Figura 2: Número de estudos classificados de acordo com o desenho.

Na tabela 1, analisamos todos os estudos que compararam modalidades de TRS, agrupando DPAC com DPA e, se no mesmo estudo houvesse dois tipos de terapia hemodialítica comparada com DP, estas foram agrupadas como HD. Exemplo: comparar HD realizada no domicílio versus HD realizada no centro versus DP, as modalidades hemodialíticas foram classificadas como HD. Avaliamos somente estudos comparativos diretos, excluímos aqueles que avaliaram o impacto de outras variáveis no custo. Exemplo: impacto do diabetes mellito no custo da HD versus DP. Os desenhos dos estudos também eram diferentes pois o objetivo desta análise foi avaliar as diferenças entre os custos em diversos

Tabela 1: Estudos comparativos entre os tipos de terapia renal substitutiva destacando àquela que apresentou menor custo.

\begin{tabular}{lcl}
\hline \multicolumn{1}{c}{ Modalidades comparadas } & Número de estudos & Menor custo \\
\hline DPAC vs DPA & 3 & DPA- 1 \\
DP vs HD & 27 & DP-20 \\
& & DP em países de alta ren- \\
& & da-1 \\
& & HD- 4 \\
& & \\
DP vs HD como primeira opção & & Sem diferença-2 \\
DP vs HD urgente & 3 & DP-3 \\
HD domiciliar vs HD convencional & 1 & DP-1 \\
HD domiciliar vs HD convencional vs HD & 2 & HD domiciliar-2 \\
hospitalar & 1 & HD convencional-1 \\
HD vs HDF & & \\
HD vs HD de alta dose & 1 & HDF-1 \\
HD vs HD noturna & 2 & HD de alta dose- 2 \\
HD vs HDF vs DP & 1 & HD noturna \\
DP vs HD vs Tx & 1 & DP-1 \\
HD vs Tx & 6 & HD-1 \\
DP vs HD vs TX vs Pré-diálise & 1 & Pré-diálise-1 \\
\hline HD - hemodiálise; DP - diálise peritoneal; HDF - hemodiafiltração; TX - transplante renal; vs - versus.
\end{tabular}




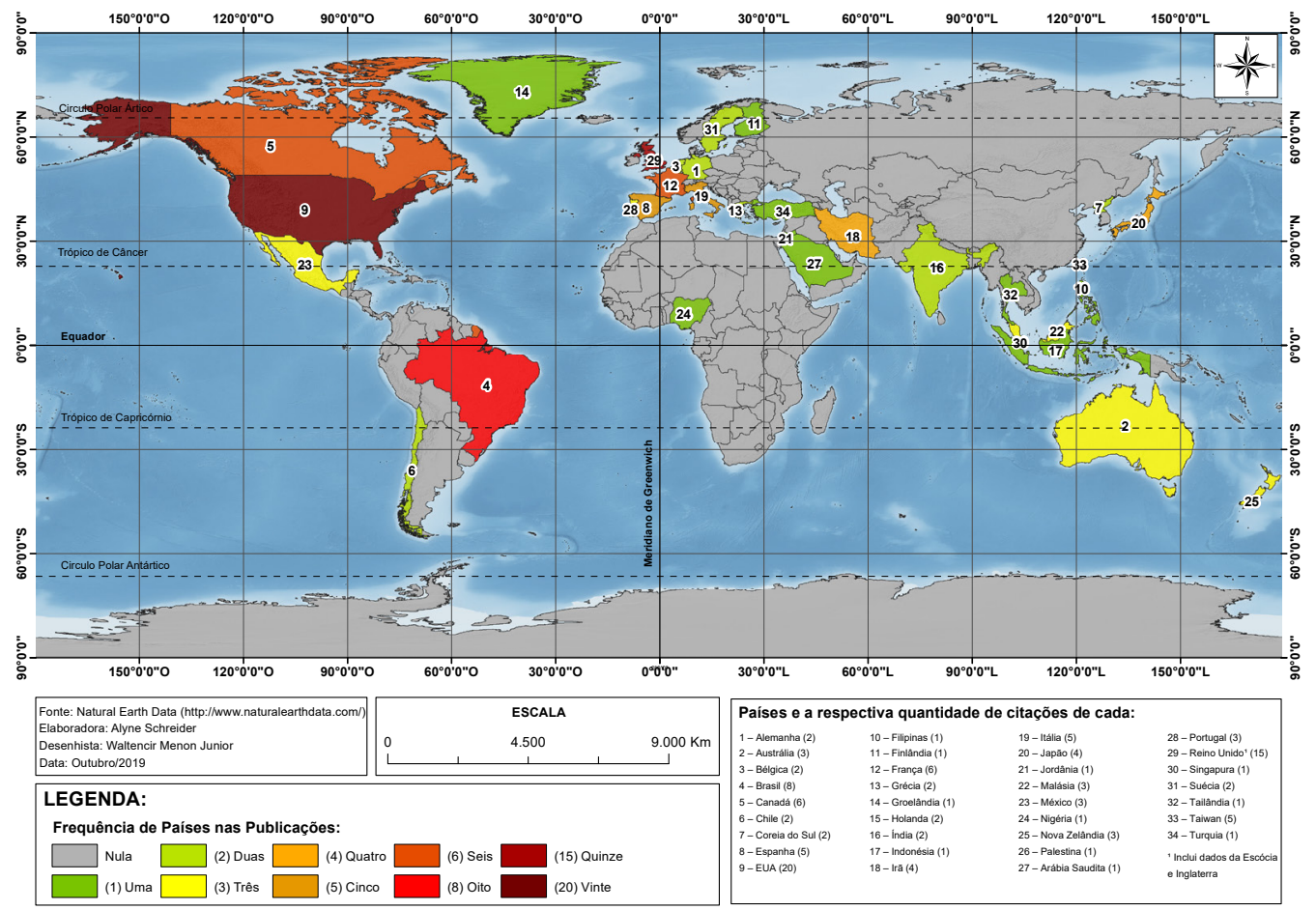

Figura 3: Estudos de custo de acordo com a localização geográfica onde foram realizados.

cenários de estudos.

\section{Diálise Peritoneal ("Peritoneal dialysis" AND "coast and cost analyses"; AND "Peritoneal dialysis" AND "health expenditures")}

Adotaremos uma abordagem cronológica e geográfica para discussão dos estudos realizados em diálise peritoneal.

A partir de 1968 começamos a encontrar estudos sobre custos em DP. ${ }^{4}$ Inicialmente esses estudos descreviam apenas que o método era factível e de baixo custo, ${ }^{4}$ além de avaliar a adequação da prescrição. ${ }^{5}$ Já nessa época eram descritas várias barreiras para a realização de diálise domiciliar. ${ }^{6}$ Entre as décadas de 1960 e 1980, os estudos de custo em DP foram escassos na literatura.

A partir da década de 1990 começam a ser realizados estudos de custo efetividade, custo utilidade, custo benefício e custo minimização em diálise peritoneal.

Em 1990, no Brasil, um estudo de custo efetividade, realizado por Sesso et $\mathrm{al}^{7}$, comparou 0 custo das modalidades de TRS, quais sejam, DPAC, HD e transplante renal com doador cadáver, concluiu que $\mathrm{HD}$ era mais custo efetiva devido ao reuso dos capilares. Neste mesmo ano, um estudo neozelandês, comparou o custo efetividade das modalidades de TRS, incluindo também hemodiálise domiciliar, e, contrariamente, concluiu que o transplante renal e a DPAC apresentaram melhor custo efetividade. ${ }^{8}$

No início da década de 1990 , surgiu uma nova modalidade de diálise peritoneal, a DPA e, em 1994, há uma análise de custo nos Estados Unidos (EUA) que compara DPAC e DPA concluindo que as mesmas são equivalentes na prescrição de 9 litros para DPA e 8 litros para DPAC. ${ }^{9}$

O continente europeu apresenta inúmeros estudos sobre o custo da DP, inicialmente com metodologia pouco elaborada. Na França, Chanut et al $^{10}$ realizaram um estudo descritivo de custo das diferentes modalidades de TRS e concluiu que os centros deveriam disponibilizar todas as modalidades, já Dubois referiu que DP era muito cara e apresentava inúmeras complicações. ${ }^{11}$ Os estudos supracitados apresentavam um desenho apenas descritivo e, neste mesmo país, em 1997, foi realizada uma análise de custo para estimar o benefício para saúde pública dos tipos de TRS que concluiu que DP apresentava menor custo para saúde pública. ${ }^{12}$ Conforme o tempo passa ocorrem progressos nas tecnologias e consequentemente diferença nos resultados dos estudos.

Em meados da década de 1990 começamos a observar vários estudos de custo com outras metodologias. No Canadá, foi realizada uma análise de custo comparando HD hospitalar, HD domiciliar, HD em centros satélites e DPAC e concluiu que HD domiciliar foi mais barata. ${ }^{13}$

É importante citar que as avaliações econômicas/custo devem ser elaboradas de acordo com os melhores padrões de qualidade e comunicar seus resultados com adequada clareza de pressupostos, dados e modelização. ${ }^{14}$ Ressaltamos que os estudos 
iniciais de custo em diálise peritoneal não seguiam este padrão.

A partir do final da década de 1990, vários estudos de custo efetividade foram realizados. O continente asiático é o local onde temos o maior percentual de pacientes em DP. Um estudo realizado nas Filipinas em uma coorte histórica comparando modalidades de TRS concluiu que transplante preemptivo foi mais custo efetivo, seguido pelo DPAC. ${ }^{15} \mathrm{Na}$ Malásia, com um mesmo desenho de estudo, concluiu-se que o maior custo efetivo foi HD domiciliar. ${ }^{16}$ Em 2001, um editorial descreve as barreiras econômicas para TRS na Ásia e destaca as diferenças de custo entre os diversos países. ${ }^{17}$ Ainda na Malásia, outro autor, em 2005, realizou um estudo de custo efetividade sob a perspectiva do governo e tanto DP quanto HD mostraram-se economicamente viáveis e com custos similares. ${ }^{18}$ Para avaliar o custo da inclusão da TRS e cuidados paliativos para DRC no sistema de saúde da Tailândia, Teerawttananon $\mathrm{Y}$ et $\mathrm{al}^{19}$ realizaram uma análise de custo efetividade e concluíram que DP como primeira terapia foi mais custo efetiva. Resultados semelhantes foram vistos em Taiwan. ${ }^{20} \mathrm{Na}$ Índia, encontramos apenas um estudo descritivo sobre o modelo de pagamento da DP. ${ }^{21}$ Finalmente, na China, um estudo que comparou o custo de duas prescrições para subgrupos diferentes em diálise peritoneal concluiu que não houve diferença. ${ }^{22}$ Avaliando o unitermo "health expenditure", em 2002, foi escrito editorial onde o autor avaliou a prática de DP na Ásia e mostrou que a economia do país afetava significativamente a taxa de pacientes em DP e que a mesma foi subutilizada. ${ }^{23} \mathrm{Um}$ estudo de 2015, em Taiwan, comparou pacientes com doença renal policística e pacientes sem a doença e avaliou variáveis de desfecho, entre elas o gasto, e os resultados foram semelhantes para gastos com e sem doença renal policística. ${ }^{24} \mathrm{Em} \mathrm{2017}$, outro estudo realizado em Taiwan comparando DPA versus DPAC com um seguimento de 5 anos observou que DPAC apresentava menor custo. ${ }^{25}$

Em alguns países o percentual de pacientes em DP é historicamente muito baixo, entre eles, citamos os Estados Unidos da América (EUA). Este país possui um dos maiores registros em diálise do mundo, o chamado United States Renal Data System (USRDS). Bruns et $a^{26}$ realizou uma análise de custo comparando os dados oficiais do USRDS com dados de uma unidade universitária e concluiu que o gasto da unidade era em média 9,8\% maior que os descritos oficialmente. Holley et al $^{27}$ avaliaram se o fornecimento de suprimentos para realização de terapia domiciliar resultava em economia para o sistema de saúde local, observando que havia uma economia de 147 dólares por paciente por mês quando os suprimentos eram fornecidos.

Ainda nos EUA, em 2002, no intuito de comparar as várias modalidades de TRS, mesmo após os ajustes para comorbidades, Lee et $a^{28}$ concluíram que HD domiciliar e DP apresentaram menor custo. Neste mesmo ano, um artigo de revisão sobre custo em TRS, nos EUA, demonstrou que transplante renal e HD domiciliar foram mais custo efetivos. ${ }^{29} \mathrm{O}$ fato de aparentemente a DP não se demonstrar custo efetiva, nos EUA, motivou Neil et $\mathrm{al}^{30}$ a realizarem uma análise de custo com o objetivo de descreverem os fatores que influenciam o padrão de DP nos EUA e o custo da mesma sob a perspectiva do Medcare. Interessantemente, ele observou que quanto maior o percentual de uso da DP menor o gasto com TRS pelo Medcare. Outro estudo que comparou o custo entre DP e HD em coorte retrospectiva por dois anos descreveu como conclusão que pacientes em DP apresentaram menor probabilidade de internação e, consequentemente, menor custo. ${ }^{31}$ Com relação à forma de pagamento de TRS, uma análise do gasto com DP após implementação do sistema de pagamento por pacote mostrou que este gerou uma economia para o Medcare. ${ }^{32}$ Este mesmo autor, no mesmo ano, comparou o custo do início de DP urgente versus HD urgente e mostrou que DP apresentou menor custo. ${ }^{33}$

Na Europa, a partir do ano 2000, uma análise de custo, sob a perspectiva do governo, com o objetivo de determinar o custo direto das modalidades dialíticas concluiu que, na França, aquelas modalidades de diálise domiciliar apresentaram menor custo. ${ }^{34}$ Em 2011, outro estudo francês sugeriu a necessidade de estudos econômicos para avaliação da forma de pagamento para DP. ${ }^{35}$ Em Portugal e na Espanha, estudos de custo sobre modalidades de TRS mostraram o transplante como modalidade mais custo efetiva seguido pela DP3640. Apenas um estudo espanhol, realizado por Lamas J et $\mathrm{al}^{41}$, mostrou que HD era mais custo efetiva. Entre estes estudos, o estudo de Coentrão et al $^{40}$ abordou especificamente o custo do acesso para HD e DP e concluiu que o acesso em DP tem um menor custo, mesmo comparado com o tipo de acesso para HD de menor custo, a fístula arteriovenosa (FAV) e apresentou menor taxa de complicações. Outro estudo espanhol avaliou os custos extras entre DP e HD e referiu que qualquer estudo de custo entre essas modalidades deveria levar em contar custos com transporte, uso de medicações e taxa de hospitalização. ${ }^{38}$

Um estudo de revisão realizado por Karopadi et $\mathrm{al}^{42}$ que comparava os custos de HD e DP no mundo mostrou que países desenvolvidos apresentaram DP com menor custo e em países em desenvolvimento não existia um padrão.

No Reino Unido os resultados são conflitantes. Na Escócia, a HD hospitalar foi mais custo efetiva, ${ }^{43}$ enquanto na Inglaterra a DP apresentou menor custo. ${ }^{44}$ Ainda no Reino Unido, foi realizado um estudo de custo utilidade usando modelo de Markov que concluiu que quanto maior o número de pacientes em DP menor o custo deste procedimento. ${ }^{45}$ Outro interessante estudo avaliou os custos extras e mostrou que em transplante e HD este diminuiu com o tempo, porém em DP ele se manteve constante. ${ }^{46}$

Países escandinavos como a Finlândia e a 
Suécia também avaliaram o custo das TRS. Na Finlândia não houve diferença entre HD e DPAC e após o primeiro ano o custo do transplante caiu significativamente. ${ }^{47} \mathrm{Um}$ estudo de custo utilidade, realizado por cinco anos na Suécia, mostrou-se favorável para DP. ${ }^{48}$

Existe um percentual relevante de pacientes em DP na Austrália, neste país já é fato que a DP tem um menor custo em relação a HD. Um estudo de custo minimização em DP com e sem a solução dialítica icodextrina mostrou que a mesma diminuiu o custo da DP. ${ }^{49}$

Com menor representatividade na literatura, entre os países africanos, um estudo nigeriano, mostrou que comparando DP e HD em pacientes pediátricos, a DP apresentou menor custo. ${ }^{50}$ Países árabes como o Irã também tem a DP como opção de modalidade dialítica com menor custo, como mostrou estudo de Atapour et $\mathrm{al}^{51}$.

Nos países latinos, Correa Roter, ${ }^{52}$ em editorial, descreveu as barreiras econômicas para todas as modalidades de TRS em países em desenvolvimento, focando principalmente no México. Um estudo de custo utilidade no México comparou DPA e DPAC em 2013 e concluiu que houve melhor custo utilidade em DPA. ${ }^{53}$ No Chile, temos um importante estudo de custo utilidade realizado por Pacheco et al $^{54}$ comparando HD e DP concluindo que há similaridade de custo entre os mesmos.

No Brasil, além do estudo de Sesso et al ${ }^{7}$ citado no parágrafo refente ao início da década de 90 , Sancho e Dain realizaram um estudo teórico para discutir as possibilidades factíveis para modelar estudos de custo efetividade em TRS no nosso meio, e concluíram que o modelo mais apropriado era o de árvore de decisão e Markov. ${ }^{55}$ De Abreu et al $^{56}$ compararam os custos diretos e indiretos entre HD e DP e concluíram que DP apresentou menor custo. Cherchiglia et $\mathrm{al}^{57}$ mostrou que o gasto do ponto de vista do governo brasileiro com modalidades dialíticas difere entre os estados, além de sofrerem influência da variável idade e presença de diabetes melitto, sugerindo uma reavaliação da forma de pagamento. Recente estudo brasileiro analisa o impacto econômico entre as modalidades de TRS, apesar das dificuldades metodológicas, os autores fazem uma avaliação sob a perspectiva do SUS: avaliação de valores pagos nas diferentes terapias, medicações, além de internação e concluíram que, a partir do segundo ano, transplante renal era mais custo efetivo. ${ }^{58}$

Uma revisão de uma modalidade específica de DP, a DP assistida, tem seu custo analisado por Dratwa e concluiu que existem diferenças sociais e econômicas quando comparados os países do estudo e que muito provavelmente a diferença do custo se deve aos recursos humanos. ${ }^{59}$

Um editorial escrito por Youssouf e O'Donoghue abordou qualidade e custos como falso paradigma enfatizando que a economia em saúde passa mais pela qualidade do atendimento do que pelo custo, porém, esta abordagem ainda é incipiente na literatura. ${ }^{60}$

\section{Hemodiálise ("renal dialysis" AND "coast and cost analyses"; AND "renal dialysis" AND "health expenditures")}

Neste tópico, adotaremos uma abordagem cronológica e agruparemos os estudos por objetivos em comum.

Observamos que o primeiro estudo encontrado, realizado em 1975, se refere a uma análise de custo benefício, porém não nos moldes preconizados atualmente, chegando a conclusão que esta terapia era muito dispendiosa e que a sociedade deveria olhar cuidadosamente o uso da mesma antes de estendê-la à população geral com DRC, questionando de certa forma a validade da terapia. ${ }^{61} \mathrm{~A}$ partir de então observamos que os estudos já avaliavam esta terapia dialítica incluída nas modalidades de tratamento oferecidas ao paciente com DRC terminal e encontramos estudos comparando hemodiálse hospitalar com hemodiálise em centros satélites que concluiram que ter unidades de diálise satélites diminuía o custo. ${ }^{62,63}$

Como visto na revisão anterior, nas décadas de 1970, 1980 e 1990, houve poucos estudos sobre o tema. Ruchlin escreveu um editorial avaliando de forma geral o custo sobre a doença renal terminal enfatizando o alto peso do mesmo sobre os custos de saúde. ${ }^{64}$

Comparando HD first versus PD first, Kirby e Vale em uma revisão sistemática descrevem que HD como primeira escolha foi mais custo efetiva que DPAC, ${ }^{43}$ este achado não é confirmado pela maioria dos estudos publicados em países de alta renda como descrito na literatura. ${ }^{65-70}$

Nos países que apresentam sistemas de saúde bem estruturados e que há algumas décadas já tem a diálise domiciliar oferecida a pacientes com DRC terminal, como no Canadá, um estudo realizado, comparou o custo da HD noturna com a convencional encontrando um menor custo em HD noturna. ${ }^{71}$ Nesta mesma linha, uma revisão sistemática publicada em 2003 comparou HD domiciliar e hospitalar, concluindo que o custo por Qaly é melhor na domiciliar. ${ }^{72}$

Novamente comparando HD domiciliar com HD realizada no centro, um estudo realizado nos EUA mostrou maior custo em HD realizada no centro. ${ }^{73}$ Porém, comparando HD hospitalar com a realizada no centro, HD no centro apresenta menor custo. ${ }^{74}$

O custo da terapia dialítica em idosos também é uma preocupação recorrente, um estudo realizado no Reino Unido avaliou o custo da terapia dialítica em pacientes acima de 70 anos e observou que esta foi mais dispendiosa, principalmente pelas comorbidades apresentadas por este subgrupo. ${ }^{75}$ Chen et al ${ }^{76}$ realizou um estudo de coorte que corrobora os achados do Reino 
Unido mostrando que o custo com cuidados com idosos com DRC em final de vida foi muito maior do que com a população geral. A avaliação do custo efetividade de outras terapias que aumentem a expectativa de vida, como utilização de drogas oncológicas, deve ser realizada independentemente do paciente fazer diálise. ${ }^{77}$ É fato que a sobrevida dos pacientes em TRS aumentou e, no Japão, em uma avaliação de uma coorte por três anos os autores demonstraram que HD melhora o status econômico dos idosos. ${ }^{78}$

Em populações que residem em regiões remotas como a população indígena da Austrália, Nova Zelândia e Canadá uma revisão de estudos de custo efetividade mostrou que muitas barreiras devem ser ultrapassadas para melhorar a qualidade do atendimento a esta população. ${ }^{79}$ Uma grande coorte, na Austrália e na Nova Zelândia, avaliou as consequências sociais para o paciente, família e governo gerando informações qualitativas e financeiras que possibilitam ao gestor público investigar mais adequadamente o custo da terapia dialítica naquele país. ${ }^{80}$

Outra preocupação é o custo de TRS em unidades de ensino, vários estudos analisam este tema. Na Turquia, um estudo que incluiu três unidades universitárias e uma privada, concluiu que o transplante é mais caro no primeiro ano e posteriormente apresenta menor custo quando comparado com a DPAC e HD. ${ }^{81}$ A terapia dialítica realizada em centros acadêmicos demanda maior tempo dedicado ao tratamento mesmo quando as comorbidades são semelhantes às apresentadas por pacientes com DRC em outros centros, este achado foi visto na Holanda em um estudo que envolveu 12 centros de terapia dialítica. ${ }^{82} \mathrm{~A}$ comparação entre diferentes centros na Espanha mostrou que esta variável pode interferir no custo benefício da diálise ainda que em um mesmo país. ${ }^{83}$

Em 2005, foi possível observar estudos sobre a importância das perspectivas do prestador de serviços, estudos estes que são muito raros na literatura. Um estudo europeu avaliando os custos em vários países da Europa concluiu que este desenho torna os dados mais consistentes, transparentes e comparáveis. ${ }^{84}$

A respeito do sistema organizacional do financiamento da TRS nos EUA, Hirth sugere que reformas do sistemas de financiamento e pagamentos eram urgentes no país naquela época. ${ }^{85}$ Esta mudança ocorreu em 2017 e Turenne et al ${ }^{86}$ avaliaram uma coorte dividida em 3 momentos que compreendiam os anos 2008-2009 (pré reforma), 2010 (durante), 2011-2013 (pós reforma) e observou que houve um aumento da seleção de pacientes para DP após a reforma, que o incentivo financeiro modificou a seleção de pacientes para DP, porém o número de pacientes em DP ainda persiste baixo.

Alguns estudos de simulação são utilizados principalmente para tentar prever custo efetividade de uma determinada modalidade dialítica. Lee realizou uma simulação do custo efetividade da HD diária nos EUA e mostrou que o alto custo inviabilizava este procedimento. ${ }^{87}$ A estimativa do custo da terapia renal substitutiva também foi realizada na Alemanha, em 2010, e conclui que o procedimento de diálise foi responsável por $55 \%$ do custo total seguido pela medicação, hospitalização e transporte e que este custo aumenta com a idade. ${ }^{88} \mathrm{Um}$ estudo espanhol estimou o custo da HD em vários centros e concluiu que os centros totalmente públicos apresentaram maior custo seguido por aqueles que eram somente parcialmente subsidiados pelo governo. ${ }^{89} \mathrm{Na}$ Malásia, uma simulação que investigou o impacto econômico em um período de cinco anos comparando HD com DP concluiu que o aumento na proporção de pacientes em DP pode reduzir O custo associada à diálise. ${ }^{90}$ Comparando HD e DP através do modelo de Markov de custo efetividade, a DP foi mais custo efetiva, Qaly 193 versus 207. ${ }^{91}$ Outra simulação que comparou o dialisato com água padrão e água ultrapura estimou que a utilização do dialisato com água ultrapura poderá fazer uma economia que varia entre 371 a 425 milhões de dólares por ano no EUA. ${ }^{92}$

Estudos que tem como escopo avaliar o custo do início e da manutenção da HD domiciliar mostrou que no período entre 2004 a 2007 houve queda do custo no Canadá. ${ }^{93}$ A tentativa de iniciar um novo programa de diálise na Groenlândia mostrou maior custo do que se os pacientes fossem mantidos em centros já existentes na Dinamarca. ${ }^{94}$

Muitos estudos sobre o custo direto e indireto da HD foram realizados em vários países como o Irã, Arábia Saudita, México, Bélgica e Índia. ${ }^{95-102}$ Estes estudos tem achados muito heterogêneos com grandes diferenças no custo total anual e por sessão para HD, além da grande diferença percentual na participação dos insumos no custo total. Recursos humanos também representaram custos diversos nestes países.

Sobre o encaminhamento precoce ao nefrologista, encontramos apenas um estudo e este mostrou que a referência precoce foi associada ao menor custo da TRS. ${ }^{103}$

A variabilidade do custo da HD nos diversos países é bem demonstrada. ${ }^{104,105}$ Uma revisão sistemática realizada em países de baixa e média renda mostrou que quando levado em conta o produto interno bruto (PIB) do país, a terapia dialítica foi considerada, sempre, extremamente cara. ${ }^{106} \mathrm{Um}$ estudo Jordaniano realizado sob a perspectiva do pagador de serviço mostrou que o custo da HD é alto para o país e que estudos comparativos com outras modalidade devem ser pensados. ${ }^{107} \mathrm{Em}$ países de baixa renda, Gregory conclui ser possível realizer HD sem eletricidade quando comparou 2 equipamentos, o que diminuíria o custo e tornaria possível a realização em locais remotos sem disponibilidade de energia elétrica. ${ }^{108}$ Por outro lado, em um país de alta renda como o Japão, uma análise macro e microeconômica sobre o sistema de saúde mostrou 
que deve ocorrer neste país um aumento dos gastos em relação a saúde em geral e notadamente em relação as terapias dialíticas, porém as condutas tomadas pelos gestores vem sendo adequadas. ${ }^{109}$

A comparação entre hemodiálise convencional e hemodiafiltração é realizada em diversos estudos que não apresentam consenso. No Japão, Takura et al $^{110}$ mostraram que hemodiafiltração foi mais custo efetiva, porém Mazairac et al $^{111}$, em um estudo multicêntrico, em 2013, com envolvimento de vários países, referem que hemodiafiltração tem menor custo utilidade. Ainda no Japão, Takura realiza novo estudo em 2015 que demonstrou o mesmo achado de 2013.78

A HD de alta dose e a convencional vem sendo comparadas em diversos estudos, no Reino Unido, HD de alta dose foi mais custo efetiva, ${ }^{44}$ dado corroborado em 2016 no Reino Unido, EUA e Canadá por Laplante et al $^{112}$ em uma revisão sistemática. Na Holanda, Beby et al $^{113}$ demostraram novamente que HD de alta dose é a mais custo efetiva.

Quando comparadas às diversas modalidades de TRS, vários estudos com múltiplas metodologias foram realizados. Em 2001, um estudo italiano de Tediosi et al ${ }^{114}$ mostrou que diálise em unidades satélites tinha menor custo quando comparada à diálise hospitalar no sistema de saúde italiano, ainda neste estudo os autores referem que DPAC apresentou menor custo quando comparada à HD e DPA. Este mesmo achado é compartilhado por Zambrowski na França. ${ }^{115}$ Uma revisão realizada por Ferguson et al ${ }^{116}$ comparou o custo de HD domiciliar, HD convencional, DP e Transplante renal e conclui que transplante renal apresentou menor custo seguido por DP, HD domiciliar e HD convencional. Na Palestina, ${ }^{117}$ o transplante renal no primeiro ano foi mais caro quando comparados a DP e HD. Na Suécia, a comparação de pré diálise em estágio 4 e 5 com transplante renal, 118 HD e DP demonstrou que DP apresentou menor custo resultado similar foi encontrado na Coréia por Kim et $\mathrm{al}^{119}$. Nos EUA, um estudo transversal mostrou que pacientes em pré diálise e transplantados apresentam menor custo que pacientes em terapia dialítica. ${ }^{120} \mathrm{Na}$ Itália, foi avaliado o custo médico direto e indireto da HD, hemodiafiltração, DPAC e DPA e foi observado que DPAC teve menor custo. ${ }^{121}$ Comparando o uso de medicamentos em pacientes com DRC pré dialítica e DRC em HD, observou-se que a realização de HD aumentava em cerca de 10 vezes o custo medicamentoso deste paciente. ${ }^{101}$

O destino do lixo da terapia dialítica também faz parte do escopo dos estudos de custo, porém encontramos apenas um estudo e este assunto necessita ser melhor avaliado. ${ }^{122}$

No Brasil, em 2015, um estudo transversal de uma coorte prospectiva se propôs a descrever o custo da HD e da terapia medicamentosa suplementar mostrando que houve um aumento de pacientes e de gastos no período de 2008 a $2011 .{ }^{123}$ Ainda no Brasil, estudo que avaliou custo direto comparando 3 hospitais públicos de ensino e pesquisa mostrou uma variabilidade importante entre os custos, de $\mathrm{R} \$ 35,29$ a $\mathrm{R} \$ 184,52$ por sessão de HD. ${ }^{124}$ Em 2018, para estimar o custo da DRC diabética sob a perspectiva do SUS entre 2010 e 2016 mostrou, com uma metodologia que não deixa clara em qual base de dados foi retirados os custos pré dialíticos, que o custo da DRC dialítica foi de 180 milhões de dólares por ano e a diabetes foi responsável por $22 \%$ do custo total. ${ }^{125}$

\section{CONCLUSÃO}

A DRC é uma das principais DNT, apresentando um alto custo financeiro. A saúde, infelizmente, tem um preço, o que nos obriga a realizar estudos de custo com múltiplos pontos de vista ou desenhos de estudo em diferentes locais do mundo com diferentes modelos politicos e econômicos.

Estudos de custo são recentes na literatura, incluem métodos diversos e mesmo com métodos semelhantes a descrição dos resultados não é padronizada. Observamos uma grande variabilidade dos custos entre os países e até mesmo entre os centros de diálise.

Em linhas gerais, em países de alta renda, a DPAC e a DPA apresentam menor custo quando comparadas à HD. O transplante renal após o primeiro ano apresenta menor custo quando comparado com o primeiro ano.

No Brasil, os achados não são unânimes, mas a maioria dos estudos mostra que HD apresenta menor custo que DPAC e DPA. Os desenhos são diversos e mostram, em geral, a perspectiva do pagador e não do prestador de serviços.

Esperamos progredir para uma discussão da saúde baseada em valor.

\section{AGRADECIMENTOS}

03626-12.

Agradecemos à FAPEMIG, Edital PPSUS-N APQ

\section{REFERÊNCIAS}

1. International Society of Nephrology. ISN, supported by ERA-EDTA and ASN calls for kidney disease inclusion member states UHC plan [Internet]. 2019. [Citado em 2019/07/02] Disponível em: https://www.theisn.org/news/ item/3428-theisnsupported-by-theera-edtaand-the-asnwelcomes-the-political-declaration-on-uhc.

2. Instituto Brasileiro de Geografia e Estatística (BR). Sinopse do Censo Demográfico Brasileiro: 2010 [Internet]. Rio de Janeiro: IBGE; 2011. [Citado 2017/07/02] Disponível em: https://biblioteca.ibge.gov.br/visualizacao/livros/liv49230. pdf. 
3. International Society of Nephrology. Chronic Kidney Disease [Internet]. 2019. [Citado em 2019/08/02] Disponível em: www.theisn.org/global-atlas.

4. Richardson JA, Philbin PE, Herron GR. Low-cost system of peritoneal dialysis. $\mathrm{Br}$ Med J. 1968; 4(5633):757-9. doi: 10.1136/bmj.4.5633.757-a.

5. Mani MK, Raibagi MH, Dingankar AD. The economics of peritoneal dialysis: a cost-efficiency study. Nephron. 1976; 17(2):130-5. doi: 10.1159/000180718.

6. Rae A, Craig P, Miles G. Home dialysis: its costs and problems. Can Med Assoc J. 1972; 106(12):1305.

7. Sesso R, Eisenberg JM, Stabile C, Draibe S, Ajzen H, Ramos $O$. Cost-effectiveness analysis of the treatment of end-stage renal disease in Brazil. Int J Technol Assess Health Care. 1990; 6(1):107-14. doi: 10.1017 / s0266462300008965

8. Croxson BE, Ashton T. A cost effectiveness analysis of the treatment of end stage renal failure. N Z Med J. 1990; 103(888):171-4.

9. Newman LN, Hanslik TM, Tessman M. Cost-effective atomated peritoneal dialysis for patients with average to low transport. Anna J. 1994; 21(5):271-3.

10. Chanut C, Barnouin F, Benech JM, Bouvier P, Briat C, Hecht $M$ et al. Assessment of costs of health insurance, substitution therapy in chronic renal insufficiency. Ver Epidemiol Sante Publique. 1991; 39(4):353-64.

11. Dubois JP. Legal and economics aspects of peritoneal dialysis. Nephrologie. 1995; 16(1):145-50.

12. Lebrun T, Marissal JP, Sailly JC. Measuring the economic impact of ambulatory dialysis: the case of continuous ambulatory peritoneal dialysis. Rev Epidemiol Sante Publique. 1997; 45(6): 493-507.

13. Goeree R, Manalich J, Grootendorst P, Beecroft ML, Churchill DN. Cost analysis of dialysis treatments for end-stage renal disease (ESRD). Clin Invest Med. 1995; 18(6):45564.

14. Weinstein MC, Obrien B, Hornberger J, Jackson J, Johannesson $M$, McCabe $C$ et al. Principles of good practice for decisison therapy analytic modeling in health-care evaluation: report of the ISPOR task force on good research practice-modeling studies. Value Health. 2003; 6(1):9-17. doi: 10.46/j.1524-4733.2003.00234.

15. Naidas OD, Chan-Licuanan KR, Velasco VP, Dalay CV, Bayog $D V$, Rosete-liquete RM. Cost effectiveness analysis of alternative treatments of end- stage renal disease: Philippine experience. Transplant Proc. 1998; 30(7):3116. doi: 10.1016/s0041-1345(98)00956-7.

16. Lim TO, Lim YN, Wong HS, Ahmad G, Singam TS, Morad $Z$ et al. Cost effectiveness evaluation of the Ministry of health Malaysia dialysis programme. Med J malaysia. 1999; 54(4):442-52.

17. Li PK, Chow KM. The cost barrier to peritoneal dialysis in the developing world an Asian perspective. Perit Dial Int. 2001; 3:S307-13.

18. Hooi LS, Lim TO, Goh A, Wong HS, Tan CC, Ahmad G et al. Economic evaluation of centre haemodialysis and continuous ambulatory peritoneal dialysis in Ministry of Health hospitals, Malaysia.Nephrology(carlton). 2005; 10(1):25-32. doi: 10.1111/j.1440-1797.2005.00360.

19. Teerawattananon Y, Mugford M, Tangcharoensathien V. Economic evalution of palliative management versus peritoneal dialysis and hemodialysis for end-stage renal disease: evidence for coverage decisions in Thailand. Value Health. 10(1):61-72. doi: 10.1111/j.1524-4733.2006.00145.

20. Kao TW , Chang YY , Chen PC, Hsu CC, Chang YK, Chang $\mathrm{YH}$ et al. Lifetime costs for peritoneal dialysis and hemodialysis in patients in Taiwan. Perit Dial Int. 2013; 33(6):6718. doi: $10.3747 /$ pdi.2012.00081.

21. Yoshesd NV, Georgi A, Milly M, Rajan R, Yuvaram NV. Na Indian model for cost-effective with minimal man power and economic resources. Nephrology Dialysis Trnasplantation. 2011; 26(10):3089-91. doi: https://doi.org/10.1093/ ndt/gfr353.

22. Su CY, Lu XH, Wang T. Influence of diferente payment schemes on the cllinical outcome in peritoneal dialysis patients. Perit Dial Int. 2016; 36(2):205-12. doi: 10.3747/ pdi.2014.00132

23. Lo WK. What factors contribute to differences in the practice of peritoneal dialysis between Asian countries and the west? Perit Dial Int. 2002; 22(2):249-57.

24. Yang JY, Chen L, Chao CT, Peng YS, Chiang CK, Kao TW et al. Compative study of outcomes among patients with polycystic kidney disease on hemodialysis and peritoneal dialysis. Sci Rep. 2015; 5:12816. doi: 10.1038/srep12816.

25. Tang $\mathrm{CH}$, Wu YT, Huang SY, Chen HH, Wu MJ, Hsu BG et al. Economics costs of automated and continuous ambulatory peritoneal dialysis in Taiwan: a combined survey and retrospective cohort analysis. BMJ Open. 2017; 7(3):e15067. doi: 10.1136/bmjopen-2016-015067.

26. Bruns FJ, Seddon P, Saul M, Zeidel ML. The cost of caring for end-estage kidney disease patients: an analysis based on hospital financial transaction records. J Am Soc Nephrol. 1998; 9(5):884-90.

27. Holley JL, McGuirl K, Smith S, Caswell N. Reducing a peritoneal dialysis program's cost by chaging from a verdor-provided to a program-provided system for general medical supplies: significant savings in CCPD. Am J Kidney Dis. 1998; 31(4):662-5. doi: 10.1053/ajkd.1998.v31. pm9531183. 
28. Lee H, Manns B, Taub K, Ghali WA, Dean S, Johnson D et al. Cost analysis of ongoing care of patients with end-stage renal disease: the impact of dialysis modality and dialysis access. Am J Kidney Dis. 2002; 40(3):611-22. doi: 10.1053/ ajkd.2002.34924.

29. Winkelmayer WC, Weinstein MC, Mittleman MA, Glynn RJ, Pliskin JS. Healtn economic evaluations: the special case of end-stage renal disease treatment. Med Decis Making. 2002; 22(5):417-30. doi: 10.1177/027298902236927.

30. Neil N, Guest S, Wong G, Portoghese L, Bhattacharyya $\mathrm{SK}$, Gehr $\mathrm{t}$ et al. The financial implications for medicare of greater use of peritoneal dialysis. Clin Ther. 2009; 31(4):880-8. doi: 10.1016/j.clinthera.2009.04.004.

31. Berger A, Edelsberg J, Inglese GW, Bhattacharyya SK, Oster $\mathrm{G}$. Cost comparison of peritoneal dialysis versus hemodialysis in end-stage renal disease. Am J Manag care. 2009; 18(8):509-18.

32. Liu FX, Walton SM, Leipold R, Isbell D, Golper TA. Financial implications to Medicare from changing the dialysis modality mix under the bundled prospective payment system. Perit Dial Int. 2014; 34(7):749-57. doi: 10.3747/ pdi.2013.00305.

33. Liu FX, Ghaffari A, Dhatt H, Kumar V, Balsera C, Wallace $E$ et al. Economic evaluation of urgente start peritoneal dialysis versus urgente start hemodialysis in the United States. Medicine. 2014; 93(28):e293. Doi: 10.1097/ MD. 0000000000000293.

34. Benain JP, Faller B, Briat C, Jacquelinet C, Brami M, Aoustin $M$ et al. Cost of dialysis in France. Nephrol Ther. 2007; 3(3): 96-106. doi: 0.1016/j.nephro.2007.03.001.

35. Chanliau J, Kessler M. Peritoneal dialysis for ESRD patients: financial aspects. Nephrol Ther. 2011; 7(1):32-7. doi: $10.1016 /$ j.nephro.2010.10.004.

36. Rodrigues A. Cost benefits of peritoneal dialysis in specific groups of patients. Contrib Nephrol. 2009; 163:285-291. doi: $10.1159 / 000223812$.

37. Arrieta J, Rodríguez-Carmona A, Remón C, Pérez-Fontán M, Ortega F, Sánchez Tomero JA et al. Peritoneal dialysis is the best cost-effective alternative for maintaining dialysis treatment. Nefrologia. 2011; 31(5):505-13. doi: 10.3265/ Nefrologia.pre2011.Jul.11103.

38. Lamas Barreiro JM, Alonso Suárez M, Saavedra Alonso JA, Gándara Martínez A. Costs and added value of haemodialysis and peritoneal dialysis outsourcing agreements. Nefrologia. 2011; 31(6):656-63. doi: 10.3265/Nefrologia. pre2011.

39. Rocha MJ, Ferreira S, Martins LS, Almeida H, Dias $G$, Pedroso $S$ et al. Cost analysis of renal replacement therapy by transplant in a system of bundled payment of dialysis. Clin Transplant. 2012; 26(4):529-31. doi: 10.1111/ j.1399-0012.2011.01571.

40. Coentrão LA, Araújo CS, Ribeiro CA, Dias CC, Pestana $M J$. Cost analysis of hemodialysis and peritoneal dialysis acess in incidente dialysis patients. Perit Dial Int. 2013; 33(6):662-70. doi: 10.3747/pdi.2011.00309.

41. Lamas J, Alonso M, Saavedra J, García-Trío G, Rionda M, Ameijeiras M. Costs of chornic dialysis in a public hospital: myths and realities. Nefrologia. 2001; 21(3):283-94.

42. Karopadi AN, Mason G, Rettore E, Ronco C. Cost of peritoneal dialysis and haemodialysis across the word. Nephorol Dial Transplant. 2013; 28(10):2553-69. doi: 10.1093/ndt/ gft214.

43. Kirby L , Vale G. Dialysis for end-stage disease. Determining a cost-effective approach.Int J Technol Assess Care. 2001; 17(2): 181-9. doi: 10.1017/s0266462300105045.

44. Liu FX, Treharne C, Culleton B, Crowe L, Arici M. The financial impacto $f$ increasing home-based high dose haemodialysis and peritoneal dialysis. BMC Nephrol. 2014;15:161. doi: 10.1186/1471-2369-15-161.

45. Treharne C, Liu FX, Arici M, Crowe L, Farooqui U. Peritoneal dialysis and in-centre haemodialysis: a cost-utility analysis from a UK payer perspective. Appl Health Econ Health Policy. $2014 ; 12(4): 409-20$. doi: 10.1007/s40258-014-0108-7

46. Li B, Cairns JA, Fotheringham J, Tomson CR, Forsythe $\mathrm{JL}$, Watson $\mathrm{C}$ et al. Understanding costs of care for patients on renal replacement therapy: looking beyond fixed tariffs. Nephrol Dial Transplant. 2015; 30(10):1726-34. doi: $10.1093 / \mathrm{ndt} / \mathrm{gfv} 224$

47. Salonen $T$, Reina $T$, Oksa $H$, Sintonen $H$, Pasternack A. Cost analysis of renal replacement therapies in Finland. Am J Kidney Dis. 2003; 42(6):1228-38. doi: 10.1053/j. ajkd.2003.08.024.

48. Sennfalt K, Magnusson M, Carlsson P. Comparison of hemodialysis and peritoneal dialysis-a cost-utility analysis. Perit Dial Int. 2002; 22(1):39-47.

49. Johnson DW, Vincent K, Blizzard S, Rumpsfeld M, Just P. Cost savings from peritoneal dialysis therapy time extension using icodextrin. Adv perit Dial. 2003; 19:81-5.

50. Obiagwu PN, Abdu A.peritoneal dialysis vs. Haemodialysis in the management of paediatric acute kidney injury in Kano, Nigeria: a cost analysis. Trop med Int health. 2015; 20(1)2-7. doi: 10.1111/tmi.12409.

51. Atapour A, Eshaghian A, Taheri D, Dolatkhah S. Hemodialysis versus peritoneal dialysis, which is cost-effective? Saudi J Kidney Dis Transpl. 2015; 26(5):926-5. doi: 10.4103/1319-2442.164578

52. Roter RC. The cost barrier to renal replacement therapy and peritoneal dialysis in the developing world. Perit Dial Int. $2011 ; 21(3): 314-317$. 
53. Cortés-Sanabria L, Rodríguez-Arreola $B E$, Ortiz-Juárez VR, Soto-Molina H, Pazarín-Villaseñor L, Martínez-Ramírez HR et al. Comparison of direct medical costs between automated and continuous ambulatory peritoneal dialysis. Perit Dial Int. 2013; 33(6):679-86. doi: 10.3747/ pdi.2011.00274.

54. Pacheco A, Saffie A, Torres R, Tortella C, Llanos C, Vargas $D$ et al. Cost/Utility study of peritoneal dialysis and hemodialysis in Chile. Perit Dial Int. 2007; 27(3):359-63.

55. Sancho LG, Dain S. Cost-effectiveness analysis of renal replacement therapies: how should we desing reserch on these interventions in Brazil? Cad Saúde Publica. 2018; 24(6):1279-90. doi: 10.1590/s0102-311×2008000600009

56. Abreu MM, Walker DR, Sesso RC, Ferraz MB. A cost evalution of peritoneal dialysis and hemodialysis in the treatment of end-stage renal disease in São Paulo, Brazil. Perit Dial Int. $2013 ; 33(3): 304-15$. doi: 10.3747/pdi.2011.00138.

57. Cherchiglia ML, Gomes IC, Alvares J, Guerra Júnior A, Acúrcio Fde A, Andrade EI et al. Determinants of expenditures on dialysis in the Unified National Health System, Brazil, 2000 to 2004. Cad Saude Publica. 2010; 26(8): 1627-41. doi: $10.1590 /$ s0102-311×2010000800016.

58. Gouveia DSES, Bignelli NO, Hokazono SR, Danucalov eu, Siemens TA, Meyer F et al. Analysis of economic impact between the modality of renal replacement therapy. J Bras Nefrol. 2017; 39(2):162-71. doi: 10.5935/01012800.20170019.

59. Dratwa M. Costs of home assistance for peritoneal dialysis: results of a European survey. Kidney Int Suppl. 2008; (108):S72-5. doi: 10.1038/sj.ki.5002605.

60. Youssouf S, O>Donoghue D. Quality versus costs: a false paradigma. Perit Dial Int. 2014; 34(7):692-4. doi: 10.3747/ pdi.2014.00220.

61. Buxton MJ, West RR. Cost-benefit analysis of long-term haemodialysis for chronic renal failure. Br Med J. 1975 ; 2(5967):376-9. doi:10.1136/bmj.2.5967.376.

62. Rose W, Grossöhmichen A. Dialysis costs. Wasrd-integrated dialysis. Z Urol Nephrol. 1979; 72(1):31-5.

63. Roberts SD, Maxwell DR, Gross TL. Cost-effective care of end-stage renal disease: a billion dolar question. Ann Intern Med. 1980; 92 (2 pt 1):243-8. doi: 10.7326/00034819-92-2-243.

64. Ruchlin HS. The public cost of kidney disease. Soc Work Health Care. 1984; 9(4):1-9. doi: 10.1300/J010v09n04_01.

65. Shih YC, Guo A, Just PM, Mujais S. Imapct of initial dialysis modality and modality switxhes on medicare expenditures of end-stage renal disease patients. Kidney Int. 2005; 68(1):319-29. doi: 10.1111/j.1523-1755.2005.00413.x.
66. Baboolal K, McEwan P, Sondhi S, Spiewanowski P, Wechowski $\mathrm{J}$, Wilson $\mathrm{K}$. The cost of renal dialysis in a UK setting-a multicentre study. Nephrol Dial Transplant. 2008; 23(6):19829. doi: $10.1093 / \mathrm{ndt} / \mathrm{gfm} 870$.

67. Liu FX, Treharne C, Arici M, Culleton B. Hight dose demodialysis versus conventional in center hemodialysis: a cost-utilitary analysis from a UK payer perspective. Value health. 2015; 18(1):17-24. doi:10.1016/j.jval.2014010.002.

68. Liu FX, Walton SM, Leipold R, Isbell D, Golper TA. Financial implications to Medicare from changing the dialysis modality mix under the bundled prospective payment system. Perit Dial Int. 2014; 34(7):749-57. doi: 10.3747/ pdi.2013.00305

69. Yang F, Lau T, Luo N. Cost-effectiveness of haemodialysis and peritoneal dialysis for patients with end-stage renal disease in Singapore. Nephrology. 2016; 21(8):669-77. doi: $10.1111 /$ nep. 12668 .

70. Chang YT, Hwang JS, Hung SY, Tsai MS, Wu JL, Sung JM et al. Cost-effectiveness of hemodialysis and peritoneal dialysis: a national cohort study with 14 years follow-up and matched for comorbidities and propensity score. Sci Rep. 2016; 27(6):30266. doi: 10.1038/srep30266.

71. McFarlane PA, Pierratos A, Redelmeier DA. Cost savings of home nocturnal versus conventional in -center hemodialysis. Kidney Int. 62(2):2216-22. doi: 10.1046/j.15231755.2002.00678.x 10.1046/j.1523-1755.2002.00678.x.

72. Mowatt G, Vale L, Perez J, Wyness L, Fraser C, MacLeod A et al. Systematic review of the effectiveness and cost-effectiveness, and economic evalution, of home versus hospital or satellite unit haemodialysis for people with ens-stage renal failure. Health Technol Assess. 2003; 7(2):1-174.

73. Ploth DW, Shepp PH, Counts C, Hutchison F. Prospective analysis of global costs for maintenance of patients with ESRD. Am J Kidney Dis. 2003; 42(1):12-21. doi: 10.1016/ s0272-6386(03)00404-9.

74. Gonzalez-Perez JG, Vale G, Stearns SC, Wordsworth S. Hemodialysis for end-stage renal disease: a cost-effectiveness analysis of treatment-options. Int Technol Assess Health Care. 2005; 21(1):32-9. doi: 10.1017/ s026646230505004x.

75. Grun RP, Constantinovici N, Normand C, Lamping DL. Costs of dialysis for elderly people in the UK. Nephrol Dial Transplant. 2003; 18(10):2122-7. doi: 10.1093/ndt/gfg354.

76. Chen B, Fan VY, Chou YJ, Kuo CC. Costs of care at the end of life among elderly patients with chronic kidney disease: patterns and predictors in a nation wide cohort study. BMC Nephrol. 2017; 18(1):36. doi: 10.1186/s12882-017-04562.

77. Grima DT, Bernard LM, Dunn ES, McFarlane PA, Mendels- 
sohn DC. Cost-effectiveness analysis of therapies for chronic kidney disease patients on dialysis: a case for excluding dialysis costs. Pharmacoeconomics. 2012; 30(11):981-9. doi: 10.2165/11599390-000000000-00000.

78. Takura T. Coat-effectiveness of hemodialysis in Japan. Contrib Nephrol. 2015; 185:124-31. doi: 10.1159/000380976

79. Reilly R, Evans K, Gomersall J, Gorham G, Peters MD, Warren $S$. Effectiveness, cost effectiveness, acceptability and implementation barriers/enablers of chronic kidney disease management programs for Indigenous people in Australia, New Zealand and Canada: a systematic review of mixed evidence. BMC Health Serv Res. 2016; 6;16:119. doi: 10.1186/s12913-016-1363-0.

80. Gorham G, Howard K, Togni S, Lawton P, Hughes J, Majoni SW et al. Economic and quality of care evaluation of dialysis servisse models in remote Australia: a protocol for a mixed methods study. BMC Health Serv Res. 2017; 17(1):320. doi: 10.1186/s12913-017-2273-5.

81. Erek E, Sever MS, Akoglu E, Sariyar H, Bozfakioglu $\mathrm{S}$, Apaydin $\mathrm{S}$ et al. Cost of renal replacement therapy in Turkey. Nephrology. 2004; 9(1):33-8.

82. Kleijn R, Uyl-de Groot C, Hagen C, Diepenbroek A, Pasker-de Jong $P$, Ter Wee P. Differences in care burden of patients undergoing dialysis in diferente centres in the netherlands. J Ren Care. 2017; 43(2):98-107. doi: 10.1111/ jorc. 12193.

83. Parra E, Arenas MD, Alonso M, Martínez MF, Gamen Á, Aguarón $\mathrm{J}$ et al. Assessing value-based health care delivery for haemodialysis. J Eval Clin Pract. 2017; 23(3):477-85. doi: 10.1111/jep.12483.

84. Wordsworth S, Ludbrook A. Comparing costing results in across contry economic evalutions: the use of technology specific purchasing power parities. Health Econ. 2005; 14(1): 93-9. doi: 10.1002/hec.913.

85. Hirth RA. The organization and financing of kidney dialysis and transplant care in the United States of America. Int J Health Care Finance Econ. 2007; 7(4):301-18. doi: 10.1007/s10754-007-9019-6.

86. Turenne M, Baker R, Pearson J, Cogan C, Mukhopadhyay P, Cope E. Payment Reform and health disparities: changes in dialysis modality under the new Medicare dialysis payment system. Health Serv Res. 2018; 53(3):1430-57. doi: 10.1111/1475-6773.12713.

87. Lee CP, Zenios SA, Chertow GM. Cost-effectiveness of frequente in-center hemodialysis. J Am Soc Nephrol. 2008; 19(9):1792-7. doi: 10.1681/ASN.2008010001.

88. Icks A, Haastert B, Gandjour A, Chernyak N, Rathmann W, Giani G et. al. Costs of dialysis- a regional population-basec analysis. Nephrol Dial Transplant. 2010; 25(5):164752. doi: $10.1093 / \mathrm{ndt} / \mathrm{gfp} 672$.
89. Parra Moncasi $E$, Arenas Jiménez MD, Alonso $M$, Martínez MF, Gámen Pardo A, Rebollo P et al. Multicentre study of haemodialysis costs. Nefrologia. 2011; 31(3):299-307. doi: $10.3265 /$ Nefrologia.pre2011.Apr. 10813.

90. Bavanandan S, Ahmad G, Teo AH, Chen L, Liu FX. Budget Impact analysis of peritoneal dialysis versus conventional In-center hemodialysis in Malásia. Value Health Reg Issues. 2016; 9:8-14. doi: 10.1016/j.vhri.2015.06.003

91. Khoe LC, Kristin E, Masytoh LS, Herlinawaty E, Werayingyong $\mathrm{P}$ et al. Economic evalution of policy options for dialysis in end-stage renal disease patients under the universal health coverage in Indonesia. PLoS One. 2017; 12(5): e0177436. doi: 10.1371/journal.pone.0177436.

92. Upadhayay A, Susantitaphong, Jaber BL. Ultrapure versus standard dialysate: a cost-benefit analysis. Semin Dial. 2017; 30(5):398-402. doi:10.1111/sdi.12618.

93. Komenda P, Copland M, Makwana J, Djurdjev O, Sood MM, Levin A. The cost of starting and maintaining a large home hemodialysis program. Kidney Int. 2010; 77(11):1039-45. doi: 10.1038/ki.2010.37.

94. Kronborg C, Kjær T, Bech M. Cost analusis of establishing dialysis facilities for the treatment of chronic renal failure in Greenland. Int J Circumpolar Health. 2010; 69(5):470-9. doi: $10.3402 /$ ijch.v69i5.17687.

95. Mahdavi-Mazdeh M, Zamani M, Zamyadi M, Rajolani H, Tajbakhsh K, Heidary Rouchi A et al. Hemodialysis cost in Tehran, Iran. Hemodial Int. 2008; 12(4):492-8. doi: 10.1111/j.1542-4758.2008.00313.x.

96. Arefzadeh A, Lessanpezeshki M, Seifi S. The costs of hemodialysis in Iran. Saudi J Kidney Dis Transpl. 2009; 20(2):307-11.

97. AL Saran K, Sabry A. The cost of hemodialysis in a large hemodialysis center. Saudi J Kidney Dis Transpl. 2012; 23(1):78-82.

98. Durán-Arenas $L$, Avila-Palomares PD, Zendejas-Villanueva R, Vargas-Ruiz MM, Tirado-Gómez LL, López-Cervantes M. Direct cost analysis of hemodialysis units. Salud Publica Mex. 2011; 53(4):516-24.

99. Cleemput I, Laet C. Analysis of the costs of dialysis and the effects of an incentive mechanism for low-cost dialysis modalities. Health Policy. 2013; 110(2-3):172-9. doi: 10.1016/j.healthpol.2013.03.001.

100. Naoum P, Topkaroglou I, Kitsonis D, Skroumpelos A, Athanasakis $\mathrm{K}$, Iatrou $\mathrm{C}$ et al. Cost calculationd during "dire Straits": a cost-of-illness analysis of regular hemodialysis for end-stage renal disease in Greece. Int J Artif Organs. 2016 ; 39(2):87-9. doi: 10.5301/ijao.5000477

101. Ahlawat R, Tiwari $P, D^{\prime} C r u z ~ S$. Direct cost for trearing chronic kidney disease at an outpatient setting of a ter- 
tiary hospital: evidence from a cross-sectional study. Value Health Reg Issues. 2017;12:36-40. doi: 10.1016/j. vhri.2016.10.003.

102. KouKou MG, Smyrniotis VE, Arkadopoulos NF, Grapsa EI. Pd vs HD in post-economic crisis Greece-differences in patient characteristics and estimation of therapy cost. Perit Dial Int. 2017; 37(5):568-73. doi: doi:10.3747/pdi.2016.00292.

103. Lee J, Lee JP, Park JI, Hwang JH, Jang HM, Choi JY et al. Early nephrology referral reduces the economic costs among patients who start renal replacement thehrapy: a prospective cohort study in Korea. Plos one. 2014; 9(6):e99460. doi: $10.1371 /$ journal.pone.0099460.

104. Klarenbach SW, Tonelli M, Chui B, Manns BJ. Economic evalution of dialysis therapies. Nat Rev Nephrol. 2014; 10(11):644-52. doi: 10.1038/nrneph.2014.145.

105. Wang T, Abraham L, Akiba T, Blake P, Gokal R, Kim MJ et al. Peritoneal dialysis in asia in the 21st century: perspectives on and obstacles to peritoneal dialysis therapy in Asian countries. Perit Dial Int. 2002; 22(2):243-8.

106. Mushi L, Marschall P, Fleßa S. The cost of dialysis in low and middle-income countries: a systematic review. BMC Health Serv Res. 2015; 15:506. doi: 10.1186/s12913-015-11668.

107. Al-Shdaifat EA, Manaf MR. The economic burden of hemodialysis in Jordan. Indian J Med Sci. 2013; 67(5-6):103-16. doi: 10.4103/0019-5359.122734.

108. Gregory MC. Cost-effective dialysis for the developing world. Ethn Dis. 2009; 19(suppl 1):65-7.

109. Takemoto Y, Naganuma T. The economic issues of hemodialysis theraphy within the Japanese health care system. Contrib Nephrol. 2017; 189:257-261. doi:10.1159/000450819

110. Takura T, Kawanishi H, Minakuchi J, Nagake Y, Takahashi S. Cost-effectiveness analysis of on-line hemodiafiltration in Japan. Blood Purif. 2013; 35(1):85-9. doi: 10.1159/000346358.

111. Mazairac AH, Blankestijn PJ, Grooteman MP, Penne EL, Van Weerd NC, Hoedt $\mathrm{CH}$ et al. The cost-utility of haemodiasfiltration versus haemodialysis in the convective transport study. Nephrol Dial Transplant. 2013; 28(7):1865-73. doi: 10.1093/ndt/gft045.

112. Laplante S, Liu FX, Culleton B, Bernardo A, King D, Hudson $P$. The cost effectiveness of high-dose versus conventional haemodialysis: a systematic review. Appl Health Econ Health Policy. 2016; 14(2):185-93. doi: 10.1007/s40258015-0212-3.

113. Beby AT, Cornelis T, Zinck R, Liu FX. Cost-effectiveness of high dose hemodialysus in comparison to conventional in-center hemodialysis in the netherlands. Adv Ther. 2016; 33(11):2032-48. doi: 10.1007/s12325-016-0408-4.
114. Tediosi F, Bertolini G, Parazzini F, Mecca G, Garattini L. Costs analysis of dialysis modalities in Italy. Health Serv Manage Res. 2001; 14(1):9-17. doi: 10.1177/095148480101400102.

115. Zambrowski Jj. Cost of dialysis in France. Nephrol Ther. 2016; 12(Suppl 1):95-7. doi: 10.1016/j.nephro.2016.02.002.

116. Ferguson TW, Tangri N, Rigatto C, Komenda P. Cost-effective treatment modalities for reducing morbidity associated with chronic Kidney diasease. Expert Rev Pharmacoecon Outcomes Res. 2015; 15(2):243-52. doi: 10.1586/14737167.2015.1012069.

117. Younis M, Jabr S, Al-Khatib A, Forgione D, Hartmann $M$, Kisa A. A cost analysis of kidney replacement therapy options Palestine. Inquiry. 2015; 52:1-8. doi: 10.1177/0046958015573494.

118. Eriksson JK, Neovius M, Jacobson SH, Elinder CG, Hylander $\mathrm{B}$. healthcare costs in chronic kidney disease and renal replacement therapy: a population-based cohort study in Sweden. BMJ Open. 2016; 6(10):e012062. doi: 10.1136/ bmjopen-2016-012062

119. Kim SH, Jo MW, Go DS, Ryu DR, Parque J. Economic burden of chronic kidney disease in Lorea using national sample cohort. J Nephrol. 2017; 30(6):787-93. doi: 10.1007/ s40620-017-0380-3

120. Damien P, Lanham HJ, Parthasarathy M, Shah NL. Assessing key cost drivers associated with caring dor chronic kidney disease patients. BMC Health Serv Res. 2016; 16(1):690. doi: 10.1186/s12913-016-1922-4

121. Vaccaro CM, Sopranzi F. A comparison between the costs of dialysis treatment in Marche Region, Italy: Macerata and Tolentino hospitals. Ann Ist Super Sanita. 2017; 53(4):3449. doi: 10.4415/ANN_17_04_12

122. Ferraresi H, Nazha H, Vigotti FN, Pereno A, Di Giorgio L, Gatti R et al. Ecodialysis: first strategies to limit damages and reduce cots. G Ital Nefrol. 2014; 31(5):1-6.

123. Menezes FG, Barreto DV, Abreu RM, Roveda F, Pecoits Filho RF. Overview of hemodialysis treatment funded by the Brazilian Unified Health System: Ann economic perspective. J Bras Nefrol. 2015; 37(3):367-78. doi: 10.5935/01012800.20150057

124. Lima AF. Direct cost of monitoring conventional hemodialysis conducted by nursing professionals. Rev Bras Enferm. 2017; 70(2):357-63. doi: 10.1590/0034-7167-20160447

125. Gonçalves GMR, Silva EN. Cost of chronic kidney disease attributable to diabetes from the perpective of the Brazilian Unified Health System. Plos One. 2018; 13(10):1-15. doi:10.1371/journal.pone.0203992 